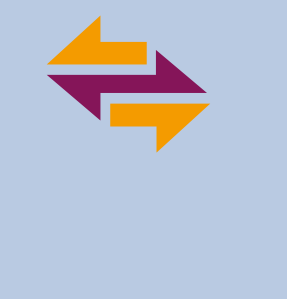

\title{
The Lehigh Presidential Nano-Human Interface Initiative: Convergence of materials and cognitive sciences
}

\author{
By Christopher J. Marvel, Joseph E. Bates, Channing E. Hambric, David A. Braun, \\ Catherine M. Arrington, and Martin P. Harmer
}

$\mathrm{R}$ ecent decades have seen rapid technological advances in the capabilities of sophisticated tools of scientific discovery used in materials research. The modern-day transmission electron microscope (TEM), for example, has been developed to image and chemically identify individual atoms (e.g., aberration-correction), ${ }^{1}$ obtain information on electron energy levels and interatomic bonding, and conduct in situ experiments among many other applications. In general, the technological advances that have introduced new data acquisition capabilities have led to widespread expansion of advanced TEMs that each cost USD\$5-7 million into the worldwide marketplace.

What has not fundamentally changed, however, is the interaction between the user and the instrument, which for the past 80 years has been mostly confined to a single operator in a tiny dark room who turns knobs and dials, and collects/visualizes/interprets data displayed on a small $\sim$ USD $\$ 200$ computer screen. It can even be argued that the interaction between human users and advanced TEMs has gotten worse over time; electron microscopes are becoming more complicated and diverse, learning curves are getting steeper, and in many cases, new microscopes are entirely encased in protective boxes that, while potentially improve image quality, limit one's ability to visualize the construction of an instrument. Overall, the latest trends of new TEM instrumentation, as well as other advanced characterization facilities, are largely overlooking the scientist and thus hampering scientific discovery!

Therefore, researchers at Lehigh University launched the Presidential Nano-Human Interfaces (NHI) Initiative to redefine the human-machine interaction between scientists and scientific instruments, which if done well, will accelerate scientific discovery. The NHI project was inspired, in part, by the competitive advantage gained by the US military who engaged cognitive scientists to study the interaction between highly trained fighter pilots and F-35 aircraft in order to devise and employ systems that minimize the cognitive load in data-rich, multitask environments, thereby improving the situational awareness of the pilot. ${ }^{2}$

The key to the previous example was the interdisciplinary collaboration between cognitive scientists and mechanical/electrical engineers, among others, to create the best product possible. Unlike traditional engineering disciplines, cognitive scientists study the structure and function of the mind as it perceives, understands, and interacts with the world. Building theoretical accounts of processes such as attention, short- and longterm memory, language, reasoning, and action are at the core of empirical and philosophical approaches to cognitive science. A recent movement in cognitive science embraces useinspired basic research; ${ }^{3}$ thus, our vision integrates the physical sciences with computer and cognitive sciences to study varying levels of expertise while operating sophisticated scientific instrumentation.

The notion of establishing interdisciplinary teams including social scientists was first recommended by the US National Academy of Engineering in which research fields like materials science would benefit from considering the cognitive skills and limitations of researchers. ${ }^{4}$ For instance, principles and insights from cognitive science can inform the design of equipment hardware and user interfaces, augment support tools for scientific reasoning and decision making, and tailor approaches to train individuals for using complex equipment. For example, Table I highlights several human-based challenges in conducting advanced electron microscopy characterization, identifies cognitive processes associated with these challenges, and recognizes recent works that speak to interventions and approaches that could be considered in addressing these specific challenges. In one case, recent interdisciplinary research of medical image perception provides an example of how the theories and methods of cognitive science can be successfully applied to guide visual search tasks in radiology, identify sources of decision error in viewing images, and support further advances in computer-aided diagnosis tools. ${ }^{3}$ Similar scenarios exist in electron microscopy as users must decide if live data are trustworthy and should be archived for future use. Overall, insights from cognitive science research have improved human 
Table I. Similarities between the scientific process of electron microscopy characterization and interests in cognitive psychology.

Challenges of Electron Microscopy Characterization

Forming and maintaining three-dimensional representations of nanoscale features

Expert microscopists switching microscope user interfaces who are trained on alternative user interfaces

Visually parse image data to identify/study anomalies

Training in electron microscopy is costly and time-consuming
Related Topics in Cognitive Science

Visual working memory, object rotation and representation, spatial reasoning

Procedural memory, sensorimotor skills, and expertise

Visual search, attention, judgment, and decision making

Skill acquisition and learning
Sample Cognitive Science Research Programs

Training of spatial skills improves object representation and mental transformation ${ }^{8}$

Experts suffer larger performance costs than novices when transitioning to a novel procedural interface for a well-learned task ${ }^{7}$

Accurate contextual information is a key factor in reasoning about and categorizing images $^{9}$

Application of principles of embodied cognition support STEM education ${ }^{10}$

STEM, science-technology-engineering-mathematics.

performance in various visual search professions.

As previously described, the mission of the NHI Initiative is to improve the human-machine interface. A part of this effort is to put the "human-in-theloop," meaning that we intend to build a system where human scientists are guided to do what they do best (e.g., think, interpret data, and be creative) while the machine completes complex tasks or initiates data analyses. So, similar to the F-35 example where the pilot can focus on the mission at hand rather than on flying the plane, the scientist will be able to focus on scientific discovery, not the operation of the microscope. The optimal human-machine interface would also allow researchers of different levels of experience to conduct experiments more intuitively, to guide each user based upon actions of prior users (i.e., an alternative form of "machine learning"), and to provide an easy system for recording metadata within an intelligent data repository. ${ }^{5}$ In many ways, this approach would render microscopy more available to the general scientist without requiring extensive training.

Another critical feature of the NHI project is the employment of modern visualization methods, particularly Microsoft's HoloLens 2 mixed reality, to collaboratively operate instruments in remote-augmented workspaces and visualize complex three-dimensional (3D) structures. Such a development will help us move from a mostly two-dimensional world that is formed by projections of $3 \mathrm{D}$ objects, into a more tangible and interactive 3D environment. This development and integration of new, human-centered capabilities is a holistic approach to engineering, ${ }^{6}$ which relies on concepts more familiar to the design community who create new user experience and user interfaces.

While our long-term goal is to disruptively change how users interact with microscopes, we must first develop a better understanding of how individuals with different levels of expertise currently interact with TEMs. Therefore, we conducted a study that employed head-mounted eye-tracking experiments of three student participants/microscopists, who had varying degrees of expertise, while they acquired atomicresolution micrographs using an aberration-corrected JEOL ARM-200CF scanning transmission electron microscope. One participant was a novice user with minimal training on the instrument and no experience in acquiring atomicresolution images, one participant was an intermediate user with extensive experience on the instrument but little experience of atomic-resolution imaging of polycrystalline materials, and one participant was an expert user who had extensive experience on the instrument as well as a deep understanding of acquiring atomic-resolution imaging of polycrystalline materials.

Participants wore a PupilLabs headmounted eye-tracking apparatus while in front of the ARM-200CF (Figure 1a), which recorded video and audio data of the participants' experience as well as eye gaze data (Figure 1b). Each participant was specifically tasked to collect atomic-resolution micrographs via one of two distinctly different user interactions: (1) a trained user interaction where the user physically interacts with knob sets in front of the microscope and navigates reciprocal space using the instrument phosphor screen; and (2) a "remote" modified user interaction where the signal is recorded on the CCD camera and shown on a computer separate from the instrument (i.e., a semi-remote setup similar to many microscopy facilities). After achieving video/audio data collection of the users on the microscope, two independent cognitive scientists parsed the time each participant spent on during the sessions and time-coded the participants' behavior into three broad tasks: navigation, where participants translated and tilted the stage to identify an area of the sample to image; focusing, where participants adjusted the focus, astigmatism, detector brightness, and detector contrast to optimize the image; and acquisition, where participants recorded and saved an image. Detailed consideration of eye gaze data can also provide insight into the visual search and decision behavior of the user, but is beyond the scope of this brief communication.

The performance time in each task of every participant and task condition was averaged over at least two or three acquired images (Figure 1c). The first 

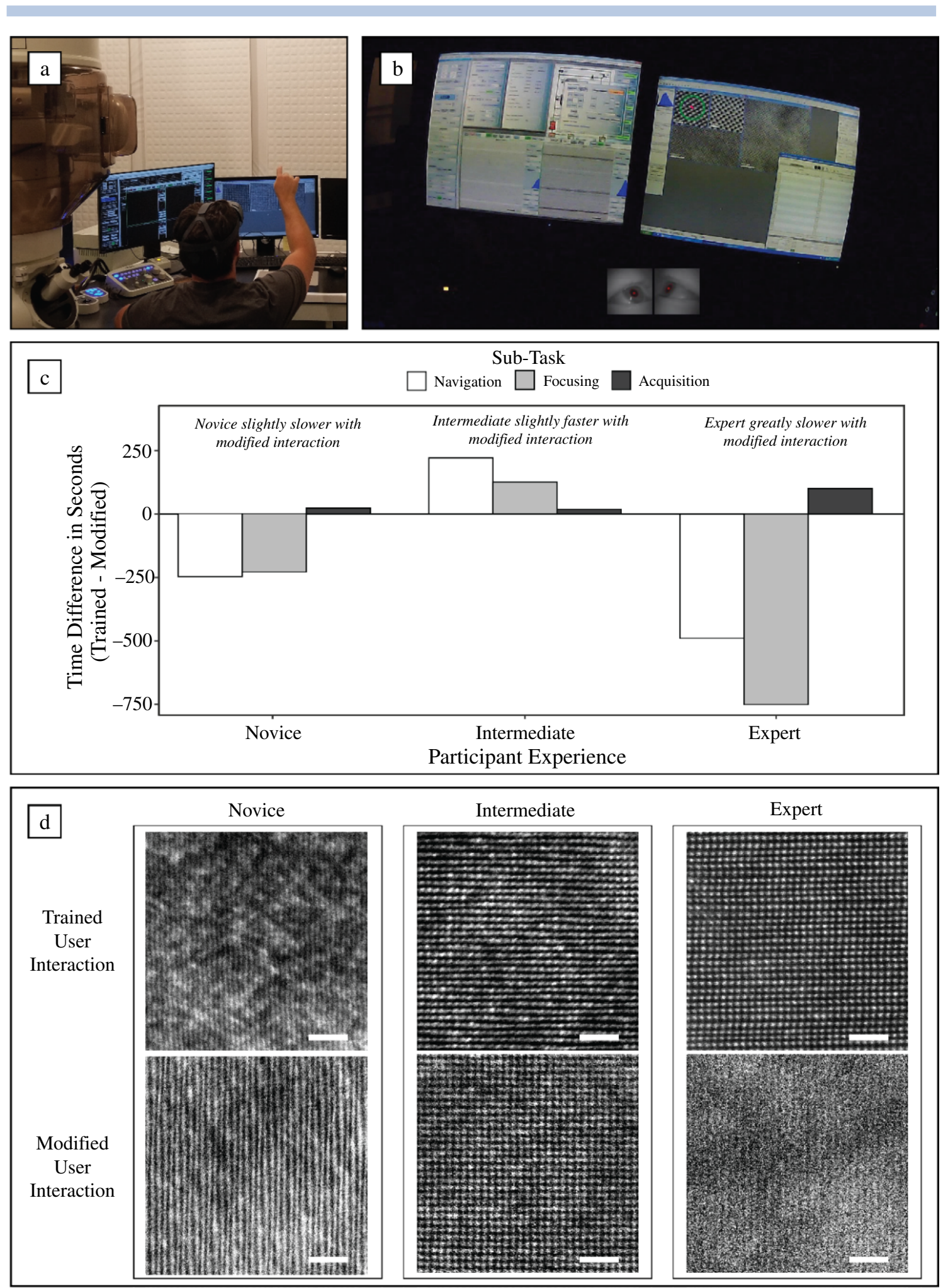

Figure 1. Eye-tracking observations of novice, intermediate, and expert users on an aberration-corrected scanning transmission electron microscope: (a) photograph of a user in front of the JEOL ARM-200CF at Lehigh University; (b) snapshot of the outward view from the eye-tracking glasses where the green circle marks the gaze point; (c) time difference between the trained and modified user interaction modes for each user during stage navigation, image focusing, and image acquisition; and (d) atomic-resolution micrographs of a nickel specimen recorded by each user via each user interaction mode (white bars are $1 \mu \mathrm{m}$ wide). 
observation was that there was little difference in acquisition time across participants and conditions, likely because acquisition is largely a function of waiting for the image to acquire and saving the image into a relevant folder. Greater variability in time on task was observed for the navigation and focusing tasks depending on the expertise of each user. Figure 1c captures the difference in performance of each participant between the trained and modified conditions. The novice microscopist required an additional $\sim 4 \mathrm{~min}$ to navigate and focus in the "remote" setting, while the intermediate microscopist required a slightly reduced time to navigate and focus in the "remote" setting. Most interestingly, the expert user was significantly hampered in the remote setting and required an additional $\sim 20$ min to complete both the navigation and focusing tasks. In fact, the expert had an overall slower performance than both the novice and intermediate participants while using the "remote" interaction. This pattern of behavior suggests that expert users already proficient in advanced TEMs will require an adjustment period prior to their being able to efficiently collect high-quality data.

Perhaps of greater interest to materials scientists is the quality of the resulting image rather than acquisition time. Therefore, performance can also be seen in the quality of the image acquired. Figure 1d shows sample images acquired by each participant under both imaging conditions. As can be seen in these examples, the expert not only took longer to acquire the image in the modified "remote" setting, but unsuccessfully achieved the same level of quality of the image as the intermediate user. It should be noted, however, that the expert did capture the best image using the trained microscope condition, so the deviation in performance can be attributed to the change in the user interface. On the other hand, the relatively poor image quality of the novice user was unchanged between the two different user settings.

The goal of this preliminary work was to study how the different microscopy user interfaces/user experiences (UIUX) affect performance for experienced versus novice microscopists. A related secondary goal was to evaluate how the eventual transition to remote microscopy impacts the efficient usage of electron microscopes. Of note, individuals with vast experience in a single user interaction environment may assume that such usability changes will not affect their performance, but counterintuitively, cognitive science research on expertise suggests that individuals with more experience may suffer greater performance disruptions than a novice user might when such transitions are required. $^{7}$

Importantly, these results suggest that switching to a novel interface, in line with the vision of NHI, might cause more performance costs for experts relative to novice and intermediate microscopists. The intermediate participant in this study was familiar with the theory of what procedures are required to collect images on this microscope, but the participant had never before used the instrument to collect images. It is possible that a computer-based interface can be more intuitive for someone in this in-between state of having some knowledge of the image collection process without having spent countless hours operating the scientific/ equipment using the trained interface. In conclusion, this brief study revealed that altering the human-machine interaction of electron microscopes will impact user performance. Therefore, as the NHI Initiative proceeds, we will be considering recent UIUX advancements to limit any performance loss of expert users.

In terms of future efforts, we are now developing/installing a state-ofthe-art observational human usability laboratory in the new Health, Science, and Technology building at Lehigh University, home to our new College of Health, which will be employed to assess operation of scientific instrumentation through mixed realities (i.e., HoloLens 2). This unique laboratory will allow more thorough exploration of the thoughts and behaviors of scientists, both individually and collaboratively, during all stages of scientific discovery and will have implications for development of 3D immersive learning environments to educate and train 21 st century scientists.

Finally, we would like to take this opportunity to suggest to the broader materials community that they consider engaging in round-robin style cognitive studies of the type described here. In order to obtain meaningful data, it is desirable to have participation from as many instrument users as possible. One way this can be accomplished is by defining a standard set of tasks and measurements to be conducted on identically prepared sample sets, and then engage multiple laboratories in the same study. Anyone interested in participating in such a round-robin study is encouraged to contact us. In addition, support of professional societies including focused workshops that discuss converging disciplines would accelerate this process, especially if including materials scientists, computer scientists, and cognitive scientists, along with STEM educators.

\section{Acknowledgments}

This work was financially supported by the Presidential Nano-Human Interfaces Initiative at Lehigh University, under the oversight of President John D. Simon and Provost Patrick V. Farrell. The authors also acknowledge Masashi Watanabe (Lehigh University), Amanda R. Krause (University of Florida) for their insightful comments during the preliminary stages of this work, and Andrea J. Harmer (Lehigh University and Kutztown University) for her leadership in Instructional Technology in the HoloLens 2 project.

\section{References}

1. D.J. Smith, Microsc. Microanal. 14, 17 (2008). https://doi.org/10.1017/S1431927608080124

2. D.G. Newman, Flying Fast Jets: Human Factors and Performance Limitations (CRC Press, Boca Raton, $\mathrm{FL}, 2017)$

3. J.M. Wolfe, Cogn. Res. Princ. Implic. 1, 17 (2016). https://doi.org/10.1186/s41235-016-0019-2 
4. National Academy of Engineering; National Academies of Sciences, Engineering, and Medicine; Division on Engineering and Physical Sciences; National Materials and Manufacturing Board; Committee on a Vision for the Future of CenterBased Multidisciplinary Engineering Research, $A$ New Vision for Center-Based Engineering Research (National Academies Press, Washington, DC, 2017)

5. D. Stansberry, S. Somnath, J. Breet, G. Shutt, M. Shankar, 2019 International Conference on Computer
Science and Computational Intelligence (CSCI) (Las Vegas, December 5-9, 2019), pp. 1312-1317

6. D. Grasso, M.B. Burkins, J.J. Helble, D. Martinelli, in Holistic Engineering Education: Beyond Technology, D. Grasso, M.B. Burkins, Eds. (Springer, New York, 2010), p.159. https://doi.org/10.1007/ 978-1-4419-1393-7

7. S.L. Beilock, S.A. Wierenga, T.H. Carr, Q. J. Exp. Psychol. A 55(4), 1211 (2002). https://doi.org/10.1080/ 02724980244000170
8. D.H. Uttal, N.G. Meadow, E. Tipton, L.L. Hand, A.R Alden, C. Warren, N.S. Newcombe, Psychol. Bull. 139(2), 352 (2013). https://doi.org/10.1037/a0028446

9. B. Fawver, J.L. Thomas, T. Drew, M.K. Mills, W.F. Auffermann, K.R. Lohse, A.M. Williams, J. Exp. Psychol. Appl. 26(4), 579 (2020). https://doi.org/10.1037/ xap0000274

10. S.M. Weisberg, N.S. Newcombe, Cogn Res. Princ. Implic. 2, 38 (2017). https://doi.org/10.1186/ s41235-017-0071-6

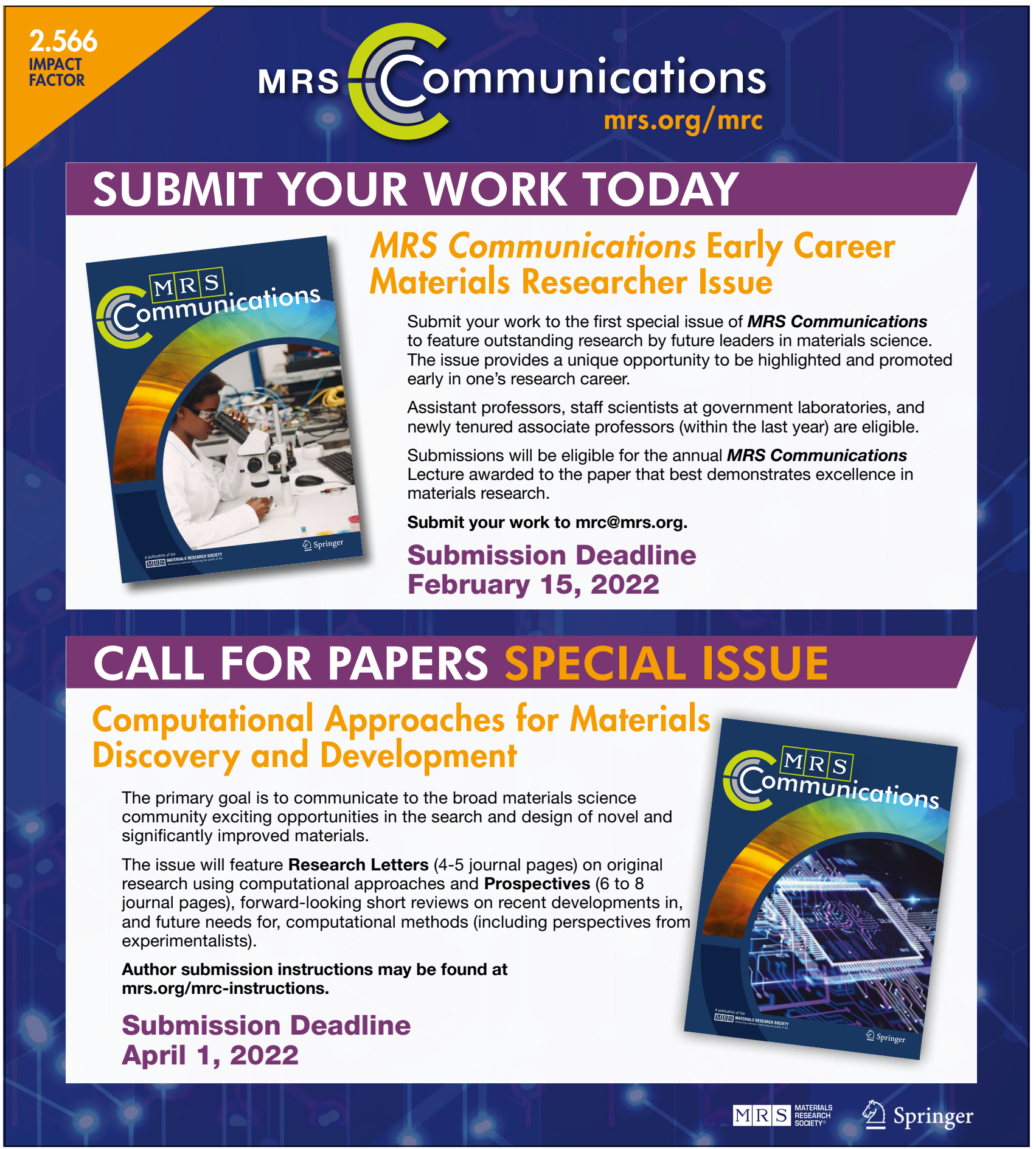

